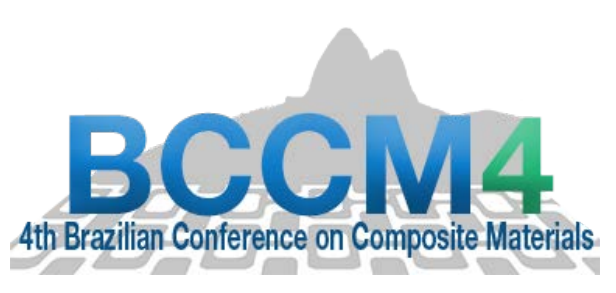

\title{
THE INFLUENCE OF MATRIX IMPREGNATION ON EFFECTIVE ELASTIC PROPERTIES OF A UNIDIRECTIONAL CARBON NANOTUBE BUNDLED COMPOSITE: A TOPOLOGY OPTIMIZATION APPROACH
}

\author{
$\underline{\text { Bruno G. Christoff }}^{(1)}$, Humberto B. Santana ${ }^{(1)}$, Eduardo L. Cardoso(2), Jandro L. Abot ${ }^{(3)}$, \\ Volnei Tita ${ }^{(1)}$ \\ (1) Department of Aeronautical Engineering, São Carlos School of Engineering, University of São \\ Paulo, São Carlos, Brazil \\ (2) Center of Technological Sciences, Santa Catarina State University, Joinville, Brazil \\ (3) Department of Mechanical Engineering, The Catholic University of America, Washington, \\ DC, USA
}

https://doi.org/10.21452/bccm4.2018.11.04

\begin{abstract}
The present work aims to find the optimum distribution of a certain amount of matrix around a pack of fibers, which is the representative volume element of a bundle of carbon nanotubes, in order to maximize a linear combination of the effective properties of the media. The homogenization by asymptotic expansion is used to find the effective properties and a topology optimization procedure is conducted to find the optimal material distribution. In the adopted Representative Volume Element (RVE), the fibers are fixed in the domain, and the optimization is performed only at matrix, whose properties are parameterized by the Solid Isotropic Material with Penalization (SIMP) method. Three distinct linear combinations of the components of the fourth order stiffness tensor are chosen, as well as three distinct admissible volume fractions for the matrix. The numerical results are presented by both the optimal material distributions in the RVEs and the convergence plots of the objective functions. The results show which regions of the RVE play a significant role for the effective properties of the composite, and may be used for a careful manufacturing process guide.
\end{abstract}

KEYWORDS: Topology optimization, Fiber reinforced composites, Imperfect adhesion, carbon nanotube bundles.

\section{INTRODUCTION}

Fiber reinforced composites play an important role in aeronautical and aerospace industries, due to its excellent specific properties. However, the behaviour of this kind of material is not 
completely understood and the manufacturing processes are often unreliable. A new state-of-theart composite like material used as reinforcing component, developed in parallel by several research groups in the past years, consists of millions of bundles of carbon nanotubes. This kind of material is very flexible, have an easy handling and have mechanical and electrical properties that can be tuned by production conditions [1]. Despite of the advantages, the mechanical, electrical and thermal properties of the bundles carbon nanotubes are highly affected by the densification and polymer impregnation [2-3].

Thus, the objective of this work lies on analyzing the effect of matrix impregnation on the effective mechanical properties of the carbon nanotubes bundles. A topology optimization approach is used in order to obtain the optimum distribution of matrix around a pack of fibers, given a pre-defined volume of matrix, in order to maximize a linear combination of the components of the homogenized fourth order stiffness tensor of the media. The concept of representative volume element (RVE) is used to represent a periodic unidirectional fiber reinforced composite, playing the role of the bundles. The homogenization by asymptotic expansion is used in order to obtain the fourth order stiffness tensor of the media and the finite element method is used to discretize the RVE domain and solve the equilibrium problems in the microscale. The topology optimization is chosen since it is the most general form of structural optimization. The fibers are kept fixed in the RVE and the optimization is performed only in the matrix. The SIMP (Solid Isotropic Material with Penalization) is used to parameterize the matrix properties and the Optimality Criteria is used to solve the optimization problem. The optimization problem is stated as the maximization of a linear combination of the components of the fourth order stiffness tensor subjected to a volume constraint.

\section{FORMULATION}

\subsection{Homogenization by asymptotic expansion}

For the determination of the effective properties of the composite, the well-known homogenization by asymptotic expansion method is used. This method is based on the assumption that a representative volume element (RVE) represents the entire domain. In other words, there is a small part of the domain in which one can find a pattern of repetition.

In the linear elasticity context, three basic considerations are adopted. The first one requires that the displacement field of the media can be written as an asymptotic expansion. The second one is that two coordinate scales are used in the analysis, one at the macroscale level (or domain scale) and the other at the microscale level (or RVE scale). These coordinate systems are related to each other by a small scalar, that in the limit tends to zero, rendering both scales in different orders of magnitude. The third one is that kinematic constraints are imposed to the boundaries of the RVE, imposing that the displacement field must have the same values on opposite faces of the RVE (periodicity).

The mathematical background is well established in literature [4-5] and states that the effective fourth order stiffness tensor of the heterogeneous media can be found by

$$
C_{i j k l}^{H}(\boldsymbol{x})=\int_{Y}\left(C_{i j k l}-C_{i j p q} \frac{\partial \chi_{p}^{k l}}{\partial y_{q}}\right) d Y
$$

where $\boldsymbol{x}$ is the coordinate vector in the macroscale, $\boldsymbol{y}$ is the coordinate vector in the microscale, $\boldsymbol{Y}$ is the vector with the dimensions of the RVE and $\chi^{k l}$ is the characteristic periodic displacement field of the RVE, given by the solution of the equilibrium equation 


$$
\int_{Y} C_{i j p q} \frac{\partial \chi_{p}^{k l}}{\partial y_{q}} \frac{\partial v_{i}}{\partial y_{j}} d Y=\int_{Y} C_{i j k l} \frac{\partial v_{i}}{\partial y_{j}} d Y
$$

where $v$ is a virtual displacement. The RVE for the present work is shown in figure 1, playing the role of a representative volume of the fiber bundles. The finite element method is used to solve Eq. 2.

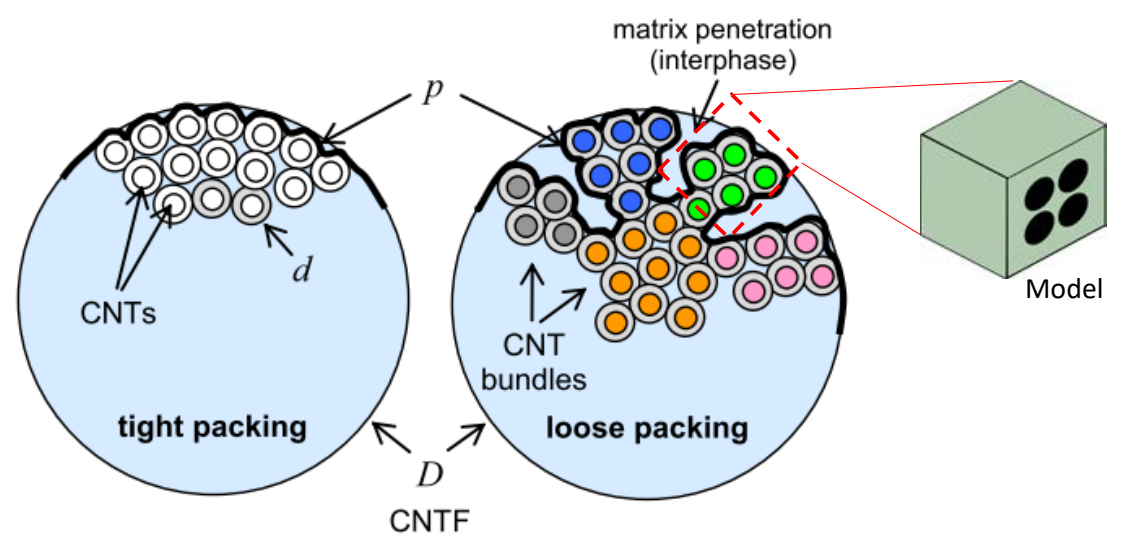

Figure 1: Illustration of Carbon Nano Tubes Fibers (CNTF) tight and loose packing and RVE model (adapted from [1])

\subsection{Material parameterization}

The main purpose of topology optimization is to determine the distribution of a set of materials within a certain fixed domain, in order to extremize an objective function, subjected to a set of constraints [6]. This is the most general form of structural optimization, allowing voids and new boundaries to be added to structure.

This work aims to find the optimal matrix distribution in the RVE in order to maximize a linear combination of the components of the homogenized fourth order stiffness tensor. A volume constraint is adopted in the matrix region, thus, a maximum matrix volume is allowed in the optimization procedure.

The design variables, for this case, play the role of the distribution of material. In a finite element method context, for each element of the mesh, a design variable, as known as relativedensity, is associated to. If the value of the design variable is 0 , there is no material associated to this particular region of the domain, conversely, if the value of the design variable is 1 , this region contains material.

Let the RVE domain $\Omega$ to be formed by two sub domains, the fiber domain $\Omega_{f}$ and the matrix domain $\Omega_{m}$. In addition, the matrix domain $\Omega_{m}$ is divided in two sub domains, the domain in which there is material $\Omega_{m}^{m a t}$ and the domain in which there is no material, $\Omega / \Omega_{m}^{m a t}$.It is considered that the fiber domain is fixed, and it is intended to find the distribution of material in $\Omega_{m}$ such that extremizes an objective function. It is desired to use the parameterization

$$
C_{i j k l}(\boldsymbol{x})=\left\{\begin{array}{rl}
C_{i j k l}^{f}, & \boldsymbol{x} \in \Omega_{f} \\
\kappa(\boldsymbol{x}) C_{i j k l}^{m}, & \boldsymbol{x} \in \Omega_{m}
\end{array},\right.
$$


where $\boldsymbol{C}$ is the fourth order stiffness tensor in each point of the domain $\Omega, \boldsymbol{C}^{f}$ and $\boldsymbol{C}^{m}$ are, respectively, the properties of fiber and matrix and $\kappa$ is a discrete function, defined in all matrix domain, given by

$$
\kappa(\boldsymbol{x})= \begin{cases}1, & \boldsymbol{x} \in \Omega_{m}^{\text {mat }} \\ 0, & \boldsymbol{x} \in \Omega / \Omega_{m}^{\text {mat }}\end{cases}
$$

This approach is ill-posed, leading to numerical problems due to the non-existence of a solution, besides the fact that the function is non-differentiable. Therefore, a relaxation of the discrete problem is necessary, which allows the design variables to assume intermediary values between 0 and 1 . However, at the end of the optimization procedure, it is desired that only discrete values remain. The SIMP method [7] is used in order to parameterize the matrix properties. Thus, the material properties in every point of domain are given by

$$
C_{i j k l}(\boldsymbol{x})=\left\{\begin{aligned}
C_{i j k l}^{f}, & \boldsymbol{x} \in \Omega_{f} \\
\rho(\boldsymbol{x})^{P} C_{i j k l}^{m}, & \boldsymbol{x} \in \Omega_{m}
\end{aligned}\right.
$$

where $\boldsymbol{\rho}$ is the vector containing the relative densities, or design variables, in every point of the matrix domain $\Omega_{\mathrm{m}}$. The relative densities are interpolated between $C_{i j k l}(\rho=0)=0$ and $C_{i j k l}(\rho=1)=C_{i j k l}^{m}$, and it is required that, at the end of the optimization procedure, all relative densities assume either 0 or 1 as their values. Also, the penalization factor is chosen as $P>1$ in order to render intermediate density values unfavorable, since the stiffness obtained is relatively small in comparison to the volume, or amount of material, involved.

\subsection{Definition of the problem}

The domain is divided in finite elements and, for every element representing the matrix, it is associated a design variable $\rho_{e}$, as known as the relative density. The objective function, $\Phi$, is defined as the linear combination of each independent component of the homogenized fourth order stiffness tensor, weighted by scalars. In addition, a volume constraint is adopted, as well as side constraints applied directly in the design variables. Thus, the optimization problem is stated as

$$
\begin{aligned}
\text { Maximize } & \Phi(\boldsymbol{\rho})=\sum_{i=1}^{21} \alpha_{i} C_{i}^{H} \\
\text { subject to } & \sum_{e=1}^{n} \rho_{e} v_{e} \leq V_{m}, \quad \rho_{e} \in \Omega_{m} \\
& \rho_{\text {min }} \leq \rho_{e} \leq 1.0
\end{aligned}
$$

where $C_{i}^{H}$ is the $i$-th independent component of the homogenized fourth order stiffness tensor, $\alpha_{i}$ is an arbitrary scalar associated to it, $V_{m}$ is the maximum allowed matrix volume, $\rho_{e}$ and $v_{e}$ are, respectively, the relative density and the volume associated to the $e$-th element of the mesh and $\rho_{\text {min }}$ is the minimum allowed value for the $e$-th design variable, chosen in order to avoid numerical problems in the solution of the linear systems.

Due to the high number of design variables associated to a topology optimization procedure, a gradient-based optimization procedure is chosen. In addition, the computational effort associated to the equilibrium is high, thus, the derivatives of both objective function and constraint are obtained analytically. Several methods can be used in order to solve a topology optimization problem and, at this work, the Optimality Criterion (OC) [6] is used. 


\section{RESULTS AND DISCUSSION}

An in-house software, written in Julia Language [8] containing all stages of the algorithm was developed. The visualization of topologies is performed in the software Gmsh [9].

For all cases, a unitary RVE is used, and the radius of each fiber is equal to 17\% of RVE length, rendering the volume fraction of fibres in the RVE of approximately $36 \%$. In addition, the domain shown in figure 1 , which reinforcement is in the direction 3 , is discretized by $60 \times 60 \times 60$ regular trilinear isoparametric hexahedral elements with incompatible modes. The mechanical properties of fiber (S-glass fiber) and matrix (steel alloy) used in the simulations are shown in table 1. It is worth mentioning that if the mechanical properties are changed during the analysis, the qualitative results remain the same. Thus, the procedure can be extended to an analysis of the carbon nanotube bundle composites.

Table 1: Mechanical properties

\begin{tabular}{cccc}
\hline$E_{f}(\mathrm{GPa})$ & $E_{m}(\mathrm{GPa})$ & $v_{f}$ & $v_{m}$ \\
\hline 303.0 & 110.3 & 0.21 & 0.3 \\
\hline
\end{tabular}

A filtering radius of 0.07 is used, applied directly to the gradient of the objective function [10] and the minimum density adopted is equal to $10^{-3}$. Three cases were chosen for the study. The objective functions of each one of the cases are given by, respectively, by $\Phi=C_{1111}^{H}+C_{2222}^{H}, \Phi=C_{1212}^{H}$ and $\Phi=C_{1313}^{H}+C_{2323}^{H}$.

In addition, for each one of the cases, matrix volume fractions of $0.25,0.50$ and 0.75 were used. Three optimization problems are defined by using equation (6) alongside the three objective functions defined above and, for each one of the optimizations problems, three distinct volume fractions are used. Thus, 9 optimizations are carried out.

The initial distribution of relative densities in the matrix is homogeneous and with the value equal to the value of the volume constraint adopted in the procedure, thus, the volume constraint is satisfied in all iterations. Figures 2 to 4 show the convergence analysis for, respectively, cases 1,2 and 3 , for a volume fraction of 0.25 . It can be noticed from figures 2 to 4 that all cases converge to a certain value of the objective function. In addition, the topologies obtained are mostly $0-1$, in other words, voids and material.

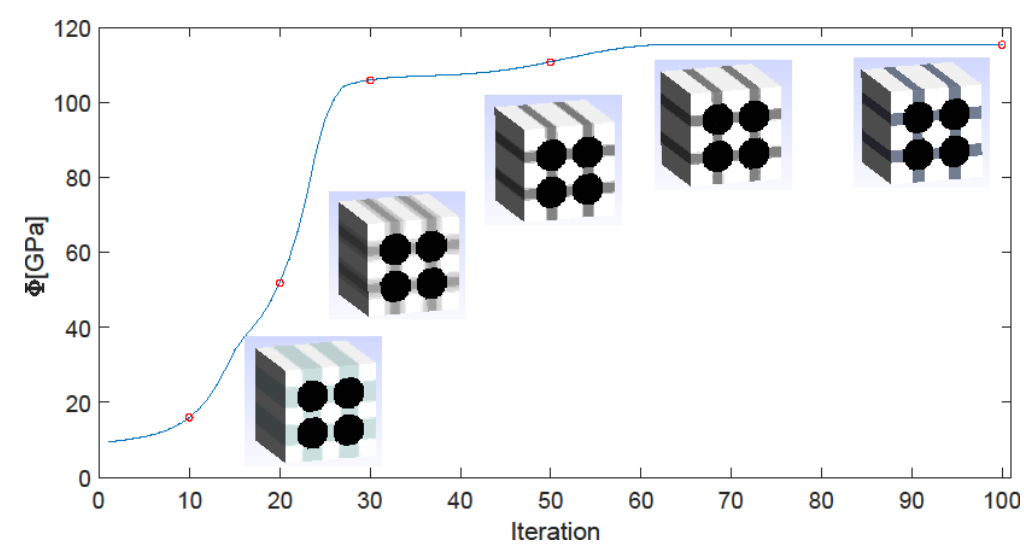

Figure 2: Convergence analysis of the objective function for case 1. Matrix volume fraction of 0.25. Topologies obtained for iterations 10, 20, 30, 50 and 100. 


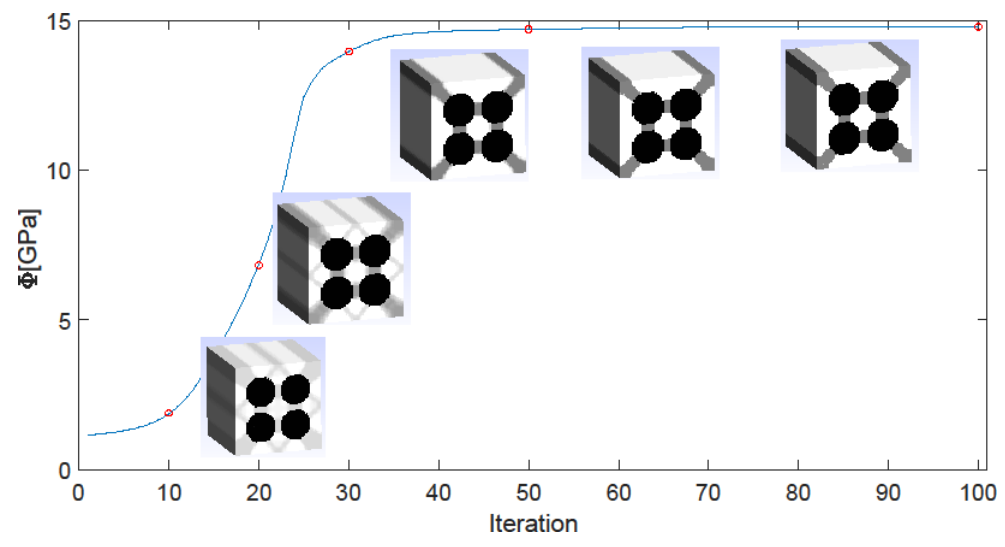

Figure 3: Convergence analysis of the objective function for case 2. Matrix volume fraction of 0.25. Topologies obtained for iterations 10, 20, 30, 50 and 100.

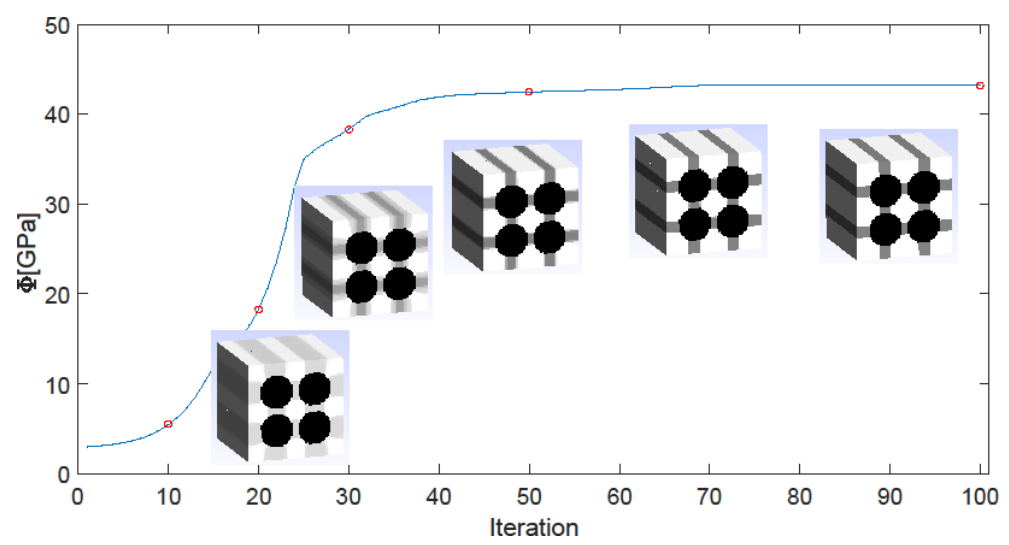

Figure 4: Convergence analysis of the objective function for case 3. Matrix volume fraction of 0.25. Topologies obtained for iterations 10, 20, 30, 50 and 100.

Only a few elements have intermediary relative densities in the final topologies. This behaviour is expected and shows that the approach adopted is consistent and that the filtering scheme is effective. Figures 5 to 7 show the optimal topologies obtained for, respectively, cases 1, 2 and 3, for volume fractions of, respectively, $0.25,0.50$ and 0.75 .
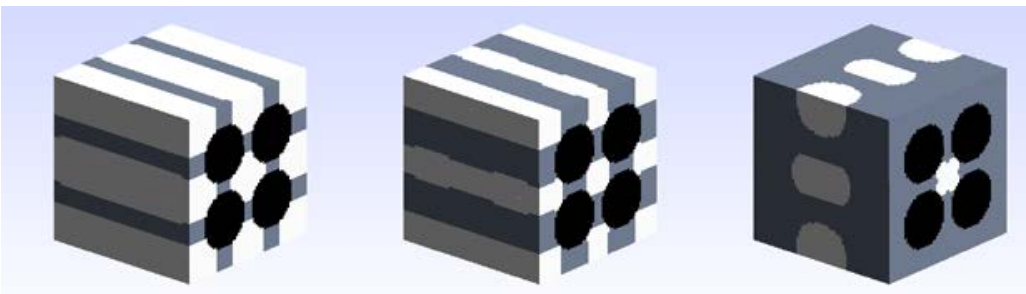

Figure 5: Optimal RVEs obtained for case 1. Matrix Volume fractions of 0.25, 0.50 and 0.75, respectively. 

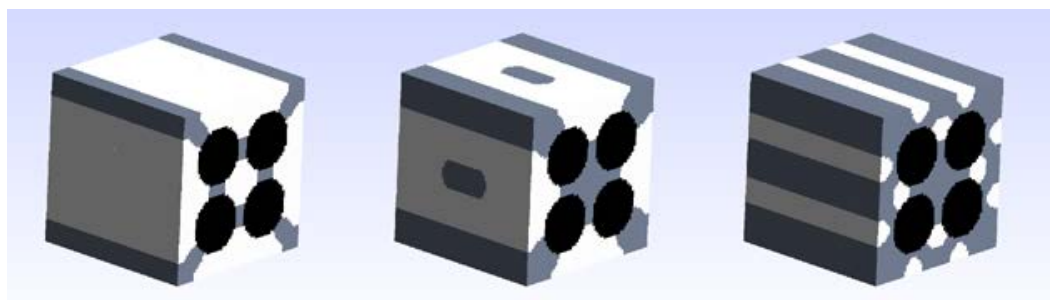

Figure 6: Optimal RVEs obtained for case 2. Matrix Volume fractions of 0.25, 0.50 and 0.75, respectively.
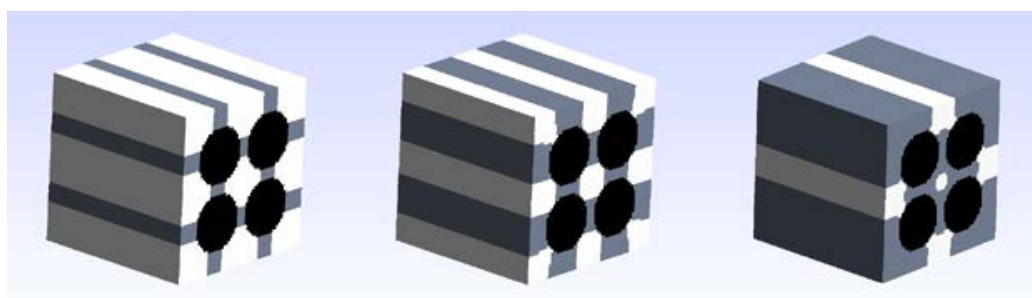

Figure 7: Optimal RVEs obtained for case 3. Matrix Volume fractions of 0.25, 0.50 and 0.75, respectively.

From figures 5 to 7 it can be seen the optimal distribution of matrix around the pack of fibers that maximizes the linear combination of the components of the homogenized fourth order stiffness tensor. The left-hand side, middle and right-hand side topologies in figures 5-7 represent, respectively, the optimal solutions for $25 \%, 50 \%$ and $75 \%$ of matrix. In the topologies with a relatively low volume of matrix, one can see the regions where the matrix has the most influence on the components of the effective stiffness tensor. For a low volume fraction, the algorithm adds material in crucial regions to maximize the objective function, shown as the dark gray regions. In the topologies with a relatively high volume of matrix, one can see the regions with the less influence in the effective properties of the media. In the topologies with a high volume fraction of matrix the white elements (lack of material) represent the regions that affect less the mechanical properties of the media.

Table 2 shows the numerical optimal results. It can be seen that a non-linear relation between the matrix volume fraction and the objective function is obtained. This is expected, since that for low volume fractions, only the most significant regions will have material and, as the volume fraction increases, regions that not play a significant role for the specific objective function will contain material as well.

Table 2: Optimal Solutions

\begin{tabular}{cccc}
\hline & $\Phi=C_{1111}^{H}+C_{2222}^{H}$ & $\Phi=C_{1212}^{H}$ & $\Phi=C_{1313}^{H}+C_{2323}^{H}$ \\
\hline & Case 1 & Case 2 & Case 3 \\
\hline$V_{m}=0.25$ & 116.2 & 14.8 & 43.3 \\
$V_{m}=0.50$ & 186.7 & 29.6 & 68.8 \\
$V_{m}=0.75$ & 288.1 & 41.8 & 93.6 \\
\hline \hline
\end{tabular}




\section{CONCLUSIONS}

In this work, numerical simulations regarding the behaviour of unidirectional fiber reinforced composites were carried out. A topology optimization approach was used in order to find the optimal distribution of a certain amount of matrix around a pack of fibers within a RVE. The results show a great consistency and that the implemented algorithm is very efficient. Also, this work can be seen as a guide in a controlled manufacturing process, since it shows the regions of matrix that most affect the effective properties of the media.

It can also be noticed that any combination of the components of the fourth order stiffness tensor can be used in the optimization. Thus, depending on the application, this approach can be used to optimize other linear combinations of the tensor. In time, this approach can be easily extended to other effective physical properties, such as thermal and electrical conductivity in composites. With the optimum topologies, an analysis regarding the effect of the matrix impregnation in a nanocomposite bundle can be made. Considering the effective fourth order tensor of the media, the loss of the properties can be foreseen with regard to the matrix impregnation and its distribution around the pack of fibers.

\section{ACKNOWLEDGEMENTS}

The authors are thankful to Coordination for the Improvement of the Higher Level Personnel (CAPES/PNPD) and National Council for Scientific and Technological Development (CNPq process number: 401170/2014-4 and 310094/2015-1).

\section{REFERENCES}

[1] Sui, Xiao Meng, Israel Greenfeld, Hagai Cohen, Xiao Hua Zhang, Qing Wen Li, and H. Daniel Wagner. "Multilevel composite using carbon nanotube fibers (CNTF)." Composites Science and Technology 137 (2016): 35-43.

[2] Vilatela, Juan J., Rupesh Khare, and Alan H. Windle. "The hierarchical structure and properties of multifunctional carbon nanotube fibre composites." Carbon 50, no. 3 (2012): 1227-1234.

[3] Jung, Yeonsu, Taehoon Kim, and Chong Rae Park. "Effect of polymer infiltration on structure and properties of carbon nanotube yarns." Carbon 88 (2015): 60-69.

[4] Sanchez-Palencia, E. "Homogenization in mechanics. A survey of solved and open problems." Rend. Sem. Mat. Univ. Politec. Torino 44, no. 1 (1986): 1-45.

[5] Hassani, Behrooz, and Ernest Hinton. "A review of homogenization and topology optimization Ihomogenization theory for media with periodic structure." Computers \& Structures 69, no. 6 (1998): 707-717.

[6] Bendsoe, Martin Philip, and Ole Sigmund. Topology optimization: theory, methods, and applications. Springer Science \& Business Media, 2013.

[7] Bendsøe, Martin Philip, and Noboru Kikuchi. "Generating optimal topologies in structural design using a homogenization method." Computer methods in applied mechanics and engineering 71, no. 2 (1988): 197-224.

[8] Bezanson, Jeff, Stefan Karpinski, Viral B. Shah, and Alan Edelman. "Julia: A fast dynamic language for technical computing." arXiv preprint arXiv:1209.5145 (2012).

[9] Geuzaine, Christophe, and Jean-François Remacle. "Gmsh: A 3-D finite element mesh generator with built-in pre-and post-processing facilities." International journal for numerical methods in engineering 79, no. 11 (2009): 1309-1331.

[10] Sigmund, Ole. "Morphology-based black and white filters for topology optimization." Structural and Multidisciplinary Optimization 33, no. 4-5 (2007): 401-424. 